\title{
UNMANNED SURFACE AND UNDERSEA VEHICLES: CAPABILITIES AND POTENTIAL
}

\author{
Vasile DOBREF, Octavian TĂRĂBUŢĂ, Cătălin-Paul CLINCI \\ “Mircea cel Bătrân” Naval Academy, Constanţa, Romania, \\ relatiipublice@anmb.ro
}

Abstract: This paper proposes a new technological solution for the sea observation using an Unmanned Surface Vehicle (USV) or Unmanned Undersea Vehicles (UUV). The term USV refers to any vehicle that operates on the surface of the water without a crew. USVs have the potential, and in some cases the demonstrated ability, to reduce risk to manned forces, provide the necessary force multiplication to accomplish military missions, perform tasks which manned vehicles cannot, and do so in a way that is affordable for the navy. In this paper we examine the use of unmanned vehicles (USV and UUV) which can replace the manned sea patrol system with additional sensors and high performance communication systems in future.

\section{Keywords: unmanned surface vehicle, unmanned undersea vehicles, robotics, navigation}

\section{Introduction}

For future naval forces the unmanned vehicles are critical components. In our days significant research and development has been performed on unmanned surface vehicles (USVs) and unmanned underwater vehicles (UUVs).

Unmanned robots have critical role during the investigation of the sea. The aim of this project is designing a robot which is capable of navigation on a given pathway, equipped with the capabilities of sending images, controlled with wireless communication. This USV navigates, avoids obstacles, using a camera, sensors for distance, humidity and temperature, wireless communications. All the hardware devices are descriptions.

\section{Description of USV}

USV is based on high-speed small craft design and unmanned systems technology to provide a highly capable unmanned surface vehicle designed for multiple missions.

DOI: 10.1515/kbo-2015-0114
The project " Grozavul " is a USV model built in order to perform certain tasks such as research, observation, transmission of real-time information (images or video), lengths and distances, atmospheric parameters: temperature, humidity, pressure.

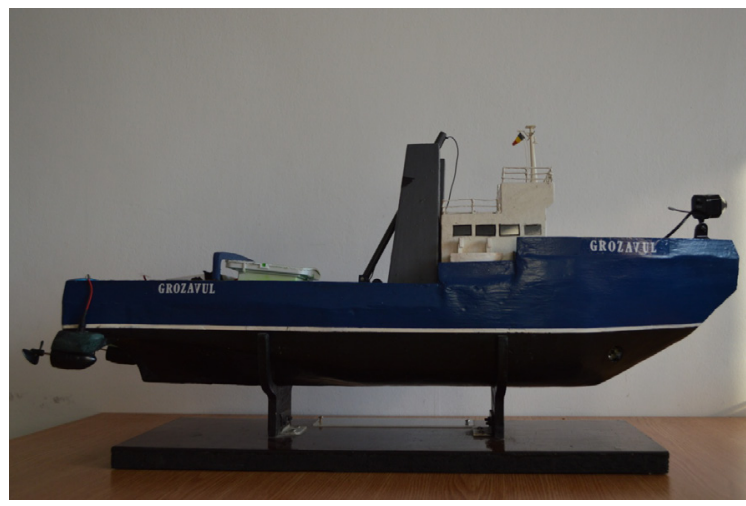

Figure 1 Project "Grozavul"

We used as a model the maritime rescue tug with the same name, and its features are: -Displacement (to): 3600 (full load )

(C) 2015. This work is licensed under the Creative Commons Attribution-NonCommercial-NoDerivatives 3.0 License. 
-Dimensions (meters: length $\mathrm{x}$ breadth $\mathrm{x}$ draft ) : $64.8 \times 14.6 \times 5.5$

-Propulsion 2 Diesel engines; $5000 \mathrm{hp}$

-Speed (Knots): 12

The scale used for the construction USVs is

1: 100. The resulted dimensions are:

- Length $65 \mathrm{~cm}$;

- Width $14 \mathrm{~cm}$;

- Height: $22 \mathrm{~cm}$;

- Displacement of $3.6 \mathrm{~kg}$;

For this project we used the following equipment: 2 electric motors operated remotely using a joystick, camera with wireless transmission, an Arduino board, sensors.

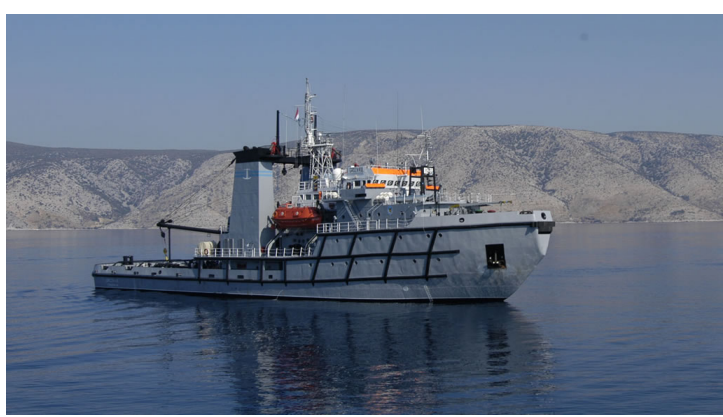

Figure 2 "Grozavul"- maritime rescue tug

\subsection{Propulsion and steering}

The model is powered by two electric motors that work in DC and it also realizes the steering. The motors are powered by batteries and joystick operated using remote control system via radio waves.

-motor power 15 watts;

-RPM 150;

-voltage 5V supply;

- autonomy 30 minutes;

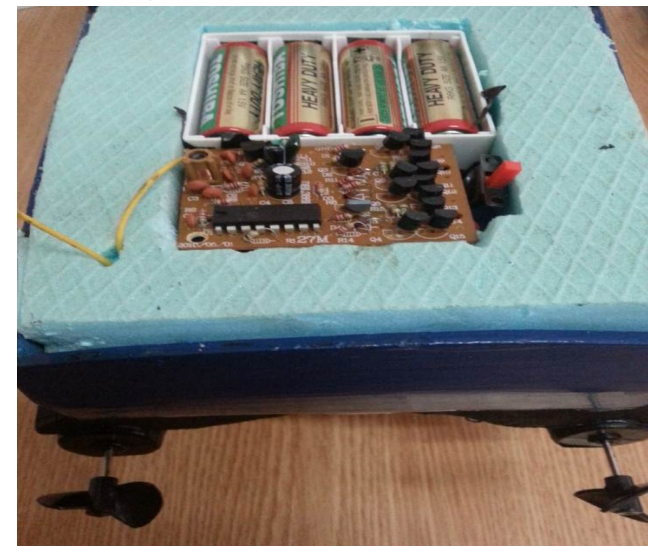

Figure 3 Arduino board, motors and batteries fitted on our model

\subsection{The camcorder}

The camera - mounted wireless video transmission in the bow and is designed to transmit video in real time using a receiver that captures information submitted to it.

Features:

-senzor 1/3 inch CMOS; -separation Ability PAL: 628x582 pixels NTSC: 510 x 492 pixels ;

-the camera has built-in microphone; -power transmission: $10 \mathrm{~mW}$; -least illumination 1,5LUX;

-voltage 8V supply;

-working temperature from -10 to +59 degrees Celsius

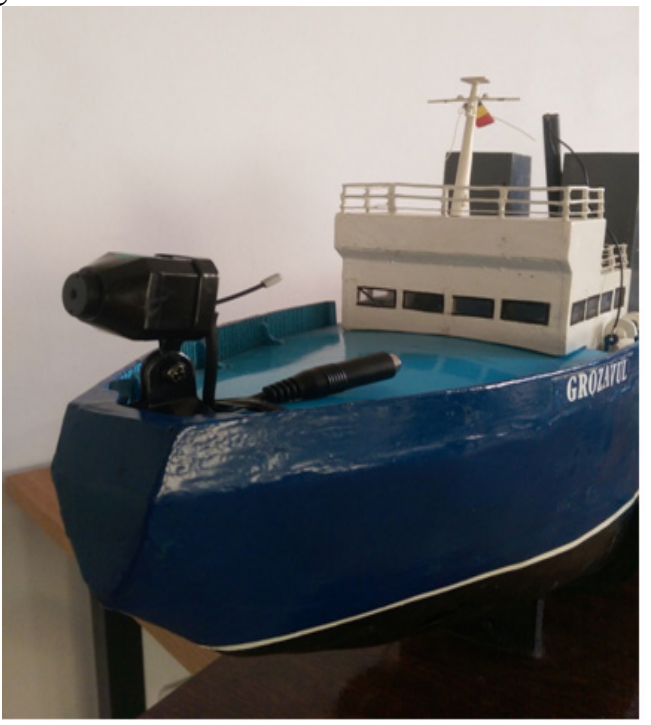

Figure 4 The camcorder

\subsection{Arduino acquisition board}

In order to control our USV, we are using an Arduino acquisition board. By this motherboard scheduling data transmitted by radio command from the command center are taken and processed, and then sent to the execution elements to achieve the desired control.

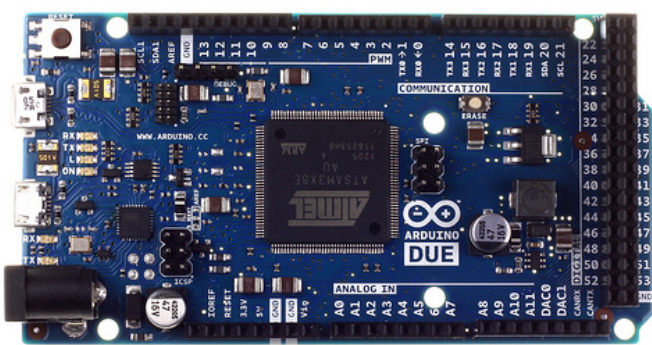

Figure 5 Arduino acquisition board Features: 
- Processor: AT91SAM3X8E;

- Working voltage: $3.3 \mathrm{~V}$;

- Supply voltage: 7-12 V;

- Digital IO pins: 54 (12 PWM);

- Analog input pin: 12;

- Memory: $512 \mathrm{~KB}$ available.

\subsection{Sensors}

Sensors represent elements which realize certain desired measurements such as: distances, air temperature, humidity and pressure etc.

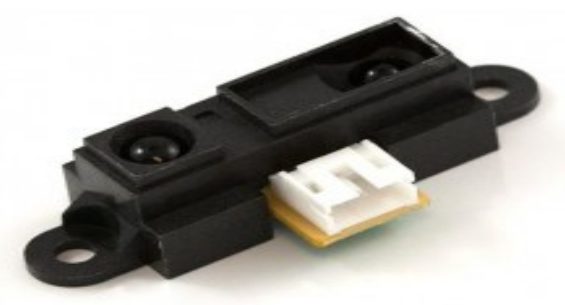

Figure 6 Digital distance sensor Sharp GP2Y0D805Z0F

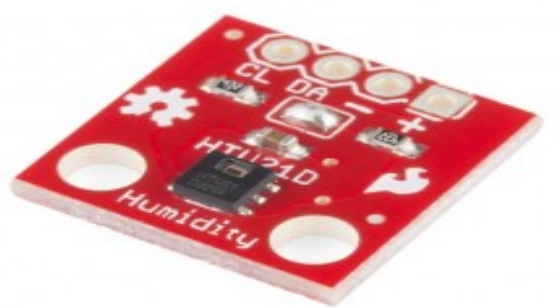

Figure 7 HTU21D humidity and temperature sensor

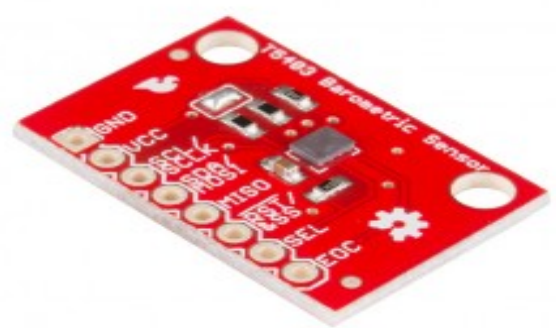

Figure 8 T5403 barometric sensor

\section{Description of UUV}

The UUV prototype is designed to carry out underwater surveillance in lakes, harbors and maritime environment, under a remote control system placed onshore or on seaborne platforms. UUV consists of a watertight streamlined body that includes the sensors, projectors and electronic microcontrollers, linked to the remote control station (laptop) via an umbilical cable that transfers data from the vehicle and commands from the operator. UUV is destined to assist divers during reconnaissance operations or to fully replace them in tough hydro-meteorological conditions.

Research and development of the prototype has been accomplished in the "Mircea cel Bătrân" Naval Academy under a contract awarded by the Romanian Naval Forces.

The necessity to use such means is given by the multiple benefits they provide, where the most important are:

- monitoring and classification of underwater objects in harbors, passes, in order to increase the safety of navigation in the present conditions of asymmetric threats facing NATO forces;

- replacement of EOD divers in their classification and neutralization of mines missions;

- increase the safety of our forces by operating at a safe distance.

The main objective for the development of a UUV prototype in the Naval Academy is to draw some conclusions about the technical and tactical capabilities necessary for future acquisitions and use of UUVs in mine-hunting operations.

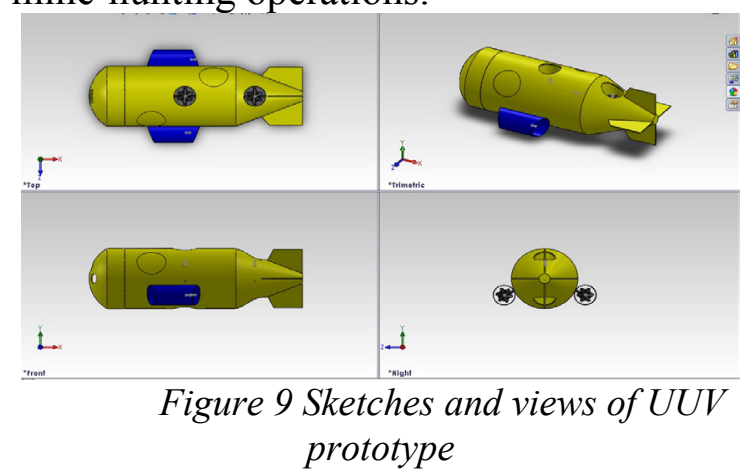




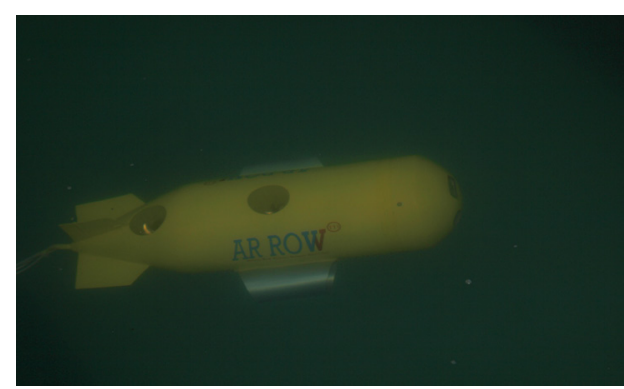

Figure 10 UUV in water

3.1 Integration of the electronic data transmission and thruster control system In order to establish the requirements for programming the remote control interface and training the UUV operator based on determined values of the dynamic characteristics and properties, we determined:

a. physical measures for input on the "acquisition board";

b. transfer functions for output measures;

c. procedures for visualization/analysis of data from sensors and for transmitting commands to the UUV.

The transmission interface of the analogue and digital signals between the remote control post and propulsion, navigation and sensors systems of the UUV prototype must take into account the nature of these signals and of the practical command necessities for vehicle movement (transfer functions).

The remote control system-vehicle interface is made by a data acquisition board, capable to process signals from sensors and signals for thruster control in accordance with the requirements of the laptop used by the operator.

Thereby, input signals into the data acquisition board will be the following:

- Video signal from the camera;

- Signal from the depth sensor;

- Signal from the space positioning sensor (accelerometer);

- Signal from the heading sensor (magnetic compass).

Effector signals, ordered by the operator are as following:

- Thrusters control in horizontal plane;

- Thrusters control in vertical plane;

- Control for projector on/off.

Transfer functions for control signals and from sensors necessary for data interpretation and controlling the UUV on a trajectory are provided by the following requirements for the interface:

- 8 single ended input channels/4 differential channels;

- 14 bits resolution;

- Sampling rate of $48 \mathrm{~kb} / \mathrm{s}$;

- 2 analogue output channels;

- 8 digital TTL type input channels;

- 8 digital TTL type output channels;

- USB interface supply.
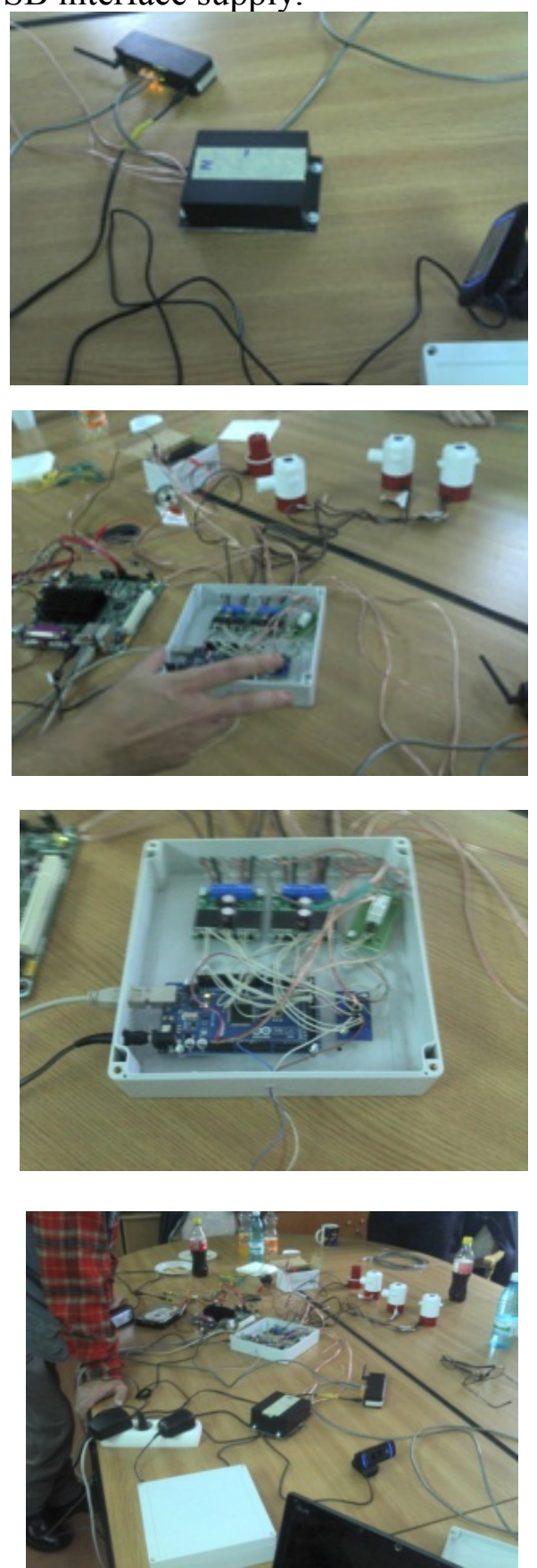

Figure 11 The UUV electronic equipments 


\section{Conclusions}

These underwater vehicles do not have onboard crew and are controlled through cable.

The information from its own sensors and controls received from an operator situated on a floatable or ground platform is transmitted through the control cable of the vehicle. UUV are generally destined for observation of the aquatic environment (maritime, fluvial or lake), search for objects in water or on the bottom and/or execution of underwater works of variable complexion.

The technical demands that the UUV should meet are:

- Design requirements;

- Requirements for deployment and tests;

- Technological requirements;

- Requirements for incorporating within the system of which is included.

Worldwide, underwater remotely operated vehicles, alongside logistic support systems are operating mainly in areas inaccessible to divers and submersibles with crew onboard. On the other hand the acquisition, maintenance and exploitation costs are far inferior compared to any other procedure for underwater works.

The UUVs have known in the last 15 years an unprecedented development, especially due to the expansion of the economic activities that benefit from them.

Main advantages in the use of UUVs are as following:

- UUVs are supplementing many of the operations that divers are executing search, observation, monitoring, retrieval of seabed objects, works on maritime installations;

- The UUVs can reach and operate in areas inaccessible to divers or to submersibles with crew onboard (oceanic trenches, pipes, tanks of toxic liquids, etc.)

- Their use significantly reduces the costs of underwater operations by diminishing personnel, equipment and required time for underwater work;

- The variety of operations made possible by UUVs confers them their versatile character, making them capable to complete various tasks, with a minimum use of effectors.

The specific objectives of the project have been fully reached, and the functional parameters are corresponding to all the operational requirements set in the project.

The fully achieved specific objectives are the following:

- The integration of sensorial and command subsystems optimum from the costs/performance ratio point of view;

- Designing the electronic remote control system through personal computer (laptop) of the UUV in accordance with self-imposed technical-tactical requirements;

- Programming of the acquisition board (interface);

- Assembly of the functional subsystems. Testing and static and dynamic evaluation, in laboratory conditions. Drawing of the dynamic diagrams;

- Tests in the pool in order to evaluate the dynamics on trajectory and the functioning of the sensor systems, cable control respectively;

- Training of the UUV operator;

- Exploitation of the results.

\section{References}

[1] Navy News Stand, Enterprise Carrier Strike Group Deploys Unmanned Surface Vehicle, Story Number: NNS031217-04, 12/17/2003.

[2] The National Defense Strategy of The United States of America, March 2005.

[3] Sea Power 21, Admiral Vern Clark, U.S. Navy. Naval Institute "Proceedings", October 2002.

[4] NAVSEAINST 3900.8A dtd 20 May 2005.

[5]Distributed Autonomous System for Maritime Domain Awareness, Douglas Horner, Naval Postgraduate School, Monterey, CA. 
[6] S. Showalter, "The Legal Status of Autonomous Underwater Vehicles," The Marine Technology Society Journal, vol. 38, no. 1, pp. 80-83, 2004.

[7] M. R. Benjamin, "Multi-objective Autonomous Vehicle Navigation inthe Presence of Cooperative and Adversarial Moving Contacts," in OCEANS 2002, Biloxi Mississippi, October 2002. 